\title{
GAC on Conjunctions of Constraints
}

\author{
George Katsirelos ${ }^{1}$ and Fahiem Bacchus ${ }^{1}$ \\ Department of Computer Science, University Of Toronto, ${ }^{\star}$ \\ Toronto, Ontario, Canada \\ [gkatsi|fbacchus]@cs.toronto.edu
}

\begin{abstract}
Applying GAC on conjunctions of constraints can lead to more powerful pruning [1]. We show that there exists a simple heuristic for deciding which constraints might be useful to conjoin. The result is a useful automatic way of improving a CSP model for GAC solving.
\end{abstract}

\section{Introduction}

Generalized arc consistency (GAC) [2] is arc consistency generalized to the non-binary case. GAC can be used as a mechanism for constraint propagation to reduce the complexity of a CSP. GAC can also be embedded in a backtracking search algorithm to provide constraint propagation dynamically (cf. the binary MAC algorithm [3, 4]).

Bessière and Régin have pointed out that GAC can also be applied to conjunctions of constraints [1], and they provide an algorithm for enforcing GAC on conjunctions. In this paper we make some further observations on this idea. Specifically, we show that there is a simple heuristic that can be used to determine whether or not it might be worthwhile grouping a collection of constraints into a conjunction and performing GAC on this conjunction.

This provides a method for achieving a potential improvement of any given CSP model automatically: using the heuristic we simply group together collections of constraints and then perform GAC on these conjunctions instead of on the individual constraints. The model with conjoined constraints can then be tested to determine if in fact an improvement has been achieved. Importantly, performing GAC on a conjunction of constraints does not require modifying the original model: the original representation of the constraints in each conjunction can be used directly by the GAC algorithm. It simply requires identifying the sets of constraints that might be worth conjoining. Since CSP modeling is a complex and potentially costly task, any improvements to a model that can be achieved automatically can help reduce the cost of finding a sufficiently efficient model. We demonstrate that this idea can be effective in two different CSP models.

\section{Background and Notation}

A CSP $(\mathcal{V}, \mathcal{C})$ consists of a set of variables $\mathcal{V}=\left\{V_{1}, \ldots, V_{n}\right\}$ and a set of constraints $\mathcal{C}=\left\{C_{1}, \ldots, C_{m}\right\}$. Each variable $V$ has a finite domain of values $\operatorname{Dom}[V]$, and can be assigned a value $v$, indicated by $V \leftarrow v$, if and only if $v \in \operatorname{Dom}[V]$. Let $\mathcal{A}$ be any set of assignments. No variable can be assigned more than one value, so $\|\mathcal{A}\| \leq n$ (i.e., the cardinality of this set is at most $n$ ). When $\|\mathcal{A}\|=n$ we call $\mathcal{A}$ a complete set of assignments. Associated with $\mathcal{A}$ is a set of variables $\operatorname{Vars} O f(\mathcal{A})$ : the set of variables assigned values in $\mathcal{A}$.

\footnotetext{
* This research was supported by the Canadian Government through their NSERC program.
} 
Each constraint $C$ is over some set of variables $\operatorname{Vars} O f(C)$, and has an arity equal to $\|\operatorname{Vars} O f(C)\|$. A constraint is a set of sets of assignments: if the arity of $C$ is $k$, then each element of $C$ is a set of $k$ assignments, one for each of the variables in $\operatorname{Vars} O f(C)$. We say that a set of assignments $\mathcal{A}$ satisfies a constraint $C$ if $\operatorname{Vars} O f(C) \subseteq \operatorname{Vars} O f(\mathcal{A})$ and there exists an element of $C$ that is a subset of $\mathcal{A}$.

$\mathcal{A}$ is said to be consistent if it satisfies all constraints $C$ such that $\operatorname{Vars} O f(C) \subseteq$ $\operatorname{Vars} O f(\mathcal{A})$, i.e., it satisfies all constraints it fully instantiates. Otherwise it is inconsistent. A solution to a CSP is a complete and consistent set of assignments.

Definition 1 (Generalized Arc Consistency). Given a constraint $C$ and a variable $V \in \operatorname{Vars} O f(C)$, a value a $\in \operatorname{Dom}[V]$ is supported in $C$ if there is a set of assignments $\mathcal{A} \in C$, such that $V \leftarrow a \in \mathcal{A}$. $\mathcal{A}$ is called a support for $\{V \leftarrow a\}$ in $C$. $C$ is (generalized) arc consistent iff each value a of each variable $V \in \operatorname{Vars} O f(C)$ is supported in C. The entire CSP is arc consistent iff each of its constraints is arc consistent.

Definition 2 (Conjunctive Consistency (Bessière and Régin)). Let $\mathcal{C}=\left\{C_{1}, \ldots, C_{k}\right\}$ be a set of constraints and Vars $O f(\mathcal{C})=\cup_{i} \operatorname{VarsOf}\left(C_{i}\right)$ be the set of variables these constraints are over. $\mathcal{C}$ is conjunctively $G A C$ iff for each value a of each variable $V \in \operatorname{VarsOf}(\mathcal{C})$ there exists a set of assignments $\mathcal{A}$ such that (1) $V \leftarrow a \in \mathcal{A}$, (2) $\operatorname{Vars} O f(\mathcal{A}) \supseteq \operatorname{Vars} O f(\mathcal{C})$, and (3) $\mathcal{A}$ satisfies each constraint $C \in \mathcal{C}$. We call such an $\mathcal{A}$ a support for $\{V \leftarrow a\}$ on the conjunction $\mathcal{C}$.

This definition says that there exists an assignment extending $V \leftarrow a$ that simultaneously satisfies all of the constraints in $\mathcal{C}$. This is equivalent to asserting that the (natural) join of all of the constraints in $\mathcal{C}$ is arc consistent (when viewed as being a new constraint relation).

As shown in [1], conjunctive GAC can be achieved in time upper bounded by $O\left(d^{\left\|\cup_{i} \operatorname{Vars} O f\left(C_{i}\right)\right\|}\right)$ where $d$ is the maximum domain size of any of the variables in $\operatorname{Vars} O f(\mathcal{C})$. Furthermore, their method does not require computing an explicit representation of the conjunction. That is, the representation of the individual constraints contained in the original model can be used directly.

Consider a CSP model containing the two constraints $C_{1}$ and $C_{2}$. If GAC is going to be used to solve the CSP, we would have to perform $\operatorname{GAC}\left(C_{1}\right)$ and $\operatorname{GAC}\left(C_{2}\right)$, a task that would require time $O\left(d^{\left\|\operatorname{Vars} O f\left(C_{1}\right)\right\|}\right)$ plus $O\left(d^{\left\|\operatorname{Vars} O f\left(C_{2}\right)\right\|}\right)$ [5]. If we choose to perform GAC on their conjunction instead, $\operatorname{GAC}\left(C_{1} \wedge C_{2}\right)$, we would need time $O\left(d^{\left\|\operatorname{Vars} O f\left(C_{1}\right) \cup \operatorname{Vars} O f\left(C_{2}\right)\right\|}\right)$. That is, the increased time required decreases as the number of variables shared by $C_{1}$ and $C_{2}$ increases. In the extreme case where $\operatorname{Vars} O f\left(C_{1}\right) \subseteq \operatorname{Vars} O f\left(C_{2}\right)$ performing GAC on the conjunction instead of over each constraint individually has the same order of complexity. In fact, for the GAC-schema algorithm of [1] it would be faster to compute $\operatorname{GAC}\left(C_{1} \wedge C_{2}\right)$ than computing both $\operatorname{GAC}\left(C_{1}\right)$ and $\operatorname{GAC}\left(C_{2}\right)$ in this case-each of these computations would require approximately the same amount of time, so we reduce the number of separate GAC computations by doing GAC on the conjunction.

From the definition it can be seen that $\operatorname{GAC}\left(C_{1} \wedge C_{2}\right)$ is a stronger consistency condition than individually enforcing $\operatorname{GAC}\left(C_{1}\right)$ and $\operatorname{GAC}\left(C_{2}\right)$. For the separate application of GAC to the two constraints, we would require only that there exists two sets of assignments $\mathcal{A}_{1}$ and $\mathcal{A}_{2}$ both extending $V \leftarrow a$, with $\mathcal{A}_{1}$ satisfying $C_{1}$ and $\mathcal{A}_{2}$ satisfying 
$C_{2}$. GAC on the conjunction requires that there exist a set of assignments $\mathcal{A}_{\wedge}$ extending $V \leftarrow a$ that satisfies $C_{1}$ and $C_{2}$ simultaneously. Hence, $\operatorname{GAC}\left(C_{1} \wedge C_{2}\right)$ has the potential to prune more values.

What is also interesting, but was not as well highlighted in [1], is that the relative strength of GAC on the conjunction increases as the number of shared variables between $C_{1}$ and $C_{2}$ increases. If these two constraints share no variables, then we can find the required set of assignments $\mathcal{A}_{\wedge}$ satisfying the conjunction, by simply unioning the two sets of assignments $\mathcal{A}_{1}$ and $\mathcal{A}_{2}$. That is, in this case GAC on the conjunction is identical to GAC on each constraint separately. On the other hand if these two constraints share $i$ variables, then the required set of assignments $\mathcal{A}_{\wedge}$ only exists if we can find an $\mathcal{A}_{1}$ satisfying $C_{1}$ and $\mathcal{A}_{2}$ satisfying $C_{2}$ that agree on their assignments to all $i$ shared variables (and then $\mathcal{A}_{\wedge}$ can again be $\mathcal{A}_{1} \cup \mathcal{A}_{2}$ ). Clearly, this constraint on the individually satisfying assignments becomes stronger as the number of shared variables, $i$, grows.

Hence, the optimal case is when $\operatorname{Vars} O f\left(C_{1}\right) \subseteq \operatorname{Vars} O f\left(C_{2}\right): \operatorname{GAC}\left(C_{1} \wedge C_{2}\right)$ is faster and it yields the maximal improved strength over doing GAC on each constraint separately.

In general, a simple heuristic for grouping constraints into conjunctive sets is as follows.

1. Initialize $C S$, the set of conjunctive sets, to contain $m$ conjunctive sets each containing the single constraint $C_{i}$.

2. If there exist two conjunctive sets $\mathcal{C}_{1}, \mathcal{C}_{2} \in C S$ such that (1) $\mathcal{C}_{1}$ and $\mathcal{C}_{2}$ share some variables, (2) the number of variables constrained by $\mathcal{C}_{1} \cup \mathcal{C}_{2}$ is less than $\max \left(\left\|\operatorname{Vars} O f\left(\mathcal{C}_{1}\right)\right\|,\left\|\operatorname{Vars} O f\left(\mathcal{C}_{2}\right)\right\|\right)+M$, and (3) also less than $N$, then remove $\mathcal{C}_{1}$ and $\mathcal{C}_{2}$ from $C S$ and add $\mathcal{C}_{1} \cup \mathcal{C}_{2}$.

3. Repeat 2 until no more such pairs exist.

In this algorithm $M$ places a limit on the increase in arity we are willing to allow, while $N$ places an absolute limit on the arity of the conjoined constraints we are willing to allow. An optimal value for $M$ will be problem dependent. For example, if $M=0$ the algorithm will only conjoin constraints satisfying $\operatorname{VarsOf}\left(C_{1}\right) \subseteq \operatorname{VarsOf}\left(C_{2}\right)$.

\section{Empirical Results}

We report on experiments we performed with two different CSP models. The first is a model for the Golomb ruler problem containing quaternary constraints developed in [6]. In this model, there is a quaternary constraint $\left|x_{i}-x_{j}\right| \neq\left|x_{k}-x_{l}\right|$ for all marks $x_{1}, \ldots, x_{m}, j<i, l<k$. Hence, over every set of four marks there will be 7 different constraints posted over these variables, 4 quaternary constraints and 3 ternary constraints. We consider finding optimal solutions and proving them to be optimal using a backtracking search that maintains GAC. For this model we set $M=0$, thus we only conjoin constraints whose variables are a subset of another.

The results are shown in Table 1. We used a 500MHz PIII machine, and the same underlying implementation for GAC on all of our tests. The first column shows the number of branches explored (and time required in CPU seconds) using the constraints without any conjunctions. The second column shows what happens when we conjoin 
$\overline{\text { Fig. } 1 \text { Backtracks and cpu time to find }(\mathrm{F}) \text { a golomb ruler of a given size or prove (P) }}$ its optimality. "-" indicates that the solver was unable to find a solution after reaching $10^{5}$ backtracks.

\begin{tabular}{|rc|rr|rr|rr|}
\hline $\begin{array}{r}\text { Problem } \\
\text { size goal }\end{array}$ & Quat $\cup$ Tern & $(\wedge$ Quat $) \cup$ Tern & $\wedge$ (Quat $\cup$ Tern $)$ \\
\hline 7 & $\mathrm{~F}$ & 95 & 0.14 & 95 & 0.07 & 90 & 0.06 \\
7 & $\mathrm{P}$ & 988 & 1.62 & 988 & 0.81 & 894 & 0.51 \\
\hline 8 & $\mathrm{~F}$ & 574 & 1.33 & 574 & 0.64 & 492 & 0.45 \\
8 & $\mathrm{P}$ & 7791 & 27.51 & 7791 & 13.35 & 7131 & 7.99 \\
\hline 9 & $\mathrm{~F}$ & 5581 & 26.51 & 5581 & 12.53 & 4920 & 7.75 \\
9 & $\mathrm{P}$ & 57545 & 403.28 & 57545 & 190.16 & 52868 & 117.16 \\
\hline 10 & $\mathrm{~F}$ & 40141 & 360.64 & 40141 & 167.45 & 36666 & 107.24 \\
10 & $\mathrm{P}$ & - & - & - & - & - & - \\
\hline
\end{tabular}

together only the 3 quaternary constraints, and the final column show what happens when we conjoin all 7 constraints (for each set of 4 variables). The results show that a useful improvement in speed is obtained and a moderate improvement in the number of branches explored. The improvement in speed is greater than the improvement in branches due to the efficiency gained by performing GAC over conjunctions (see Section 2 for a discussion of this point). The middle column demonstrates that our technique is heuristic-improved pruning is not always obtained through conjunctions. In particular, it turns out that the three quaternary constraints all logically entail each other, so enforcing one is as powerful as enforcing all three or as enforcing the conjunction. Thus, we see no improvement in number of branches. The improvement in speed arises from the fact that with conjunctions we have fewer constraints over which GAC has to be enforced.

In the second model we experiment with random 3-sat problems. These problems are converted from a set of clauses into a CSP containing a set of binary variables and a ternary constraint for each clause. In this case, we set $M=1$ and $N=4$, thus generating conjunctions over 4 variables. The results are shown in Table 2.

Fig. 2 Average number of backtracks and cpu time to prove whether a problem is satisfiable or not. The last column indicates the percentage of instances where the solver performed better if constraints were conjoined.

\begin{tabular}{|c|c|c|c|c|c|c|}
\hline \multirow{2}{*}{ \# Variables } & \# Instances & \multicolumn{2}{|c|}{ avg leafs } & \multicolumn{2}{c|}{ avg time } & \\
\hline 60 & 100 & 674.33 & 522.71 & 0.9951 & 0.8485 & 71 \\
\hline 70 & 100 & 1637.36 & 1300.21 & 2.8882 & 2.4676 & 74 \\
\hline 80 & 100 & 3504.36 & 2888.33 & 7.204 & 6.3182 & 74 \\
\hline 90 & 100 & 9204.49 & 7085.21 & 21.7589 & 17.8116 & 75 \\
\hline 100 & 100 & 19573.2 & 14434.2 & 52.5704 & 40.7247 & 83 \\
\hline
\end{tabular}


It is worthwhile noting in this case that there were instances where the solver actually performed more backtracks when using conjoined constraints than it did in the original problem. This anomaly can be attributed to the fact that 3-SAT has a special structure, which is not accounted for by the minimal remaining values-break ties by degree heuristic used. Therefore, even though the conjunctive constraints cause more pruning, they end up making the search slower by fooling the heuristic. This anomaly could probably be eliminated by using one of the heuristics that have been developed specifically for SAT problems.

If we factor out these instances, and look only at those instances where the solver performed fewer backtracks with conjunctive constraints, we find that the cpu time used is at worst only $10 \%$ more than the time used to solve the problem using the original model. This shows that despite the fact that we are generating constraints of higher arity in this case (and hence the complexity of enforcing GAC on the conjunction is higher than enforcing GAC on the individual constraints separately) the overhead of performing GAC on the conjoined constraints is alleviated by the extra pruning it generates. More specifically, Table 3 show the same results as Table 2 except that the instances where the heuristic performed poorly have been removed. The results show a useful improvement is achieved by using conjunctions.

Fig. 3 The same data Table 2 but only instances for which conjoining constraints did not interfere with the behavior of the DVO heuristic are counted.

\begin{tabular}{|c|c|c|c|c|c|c|}
\hline \multirow{2}{*}{ \# Variables } & & \multicolumn{2}{|c|}{ avg leafs } & \multicolumn{2}{c|}{ avg time } & \\
\hline 60 & 79 & 1602.62 & 540.81 & 2.35886 & 0.879747 & 88.6076 \\
\hline 70 & 78 & 3890.79 & 1322.47 & 6.84756 & 2.50756 & 93.5897 \\
\hline 80 & 82 & 7829.66 & 2706.33 & 16.0824 & 5.94561 & 90.2439 \\
\hline 90 & 79 & 21926.1 & 7214.34 & 51.7696 & 18.1687 & 94.9367 \\
\hline 100 & 85 & 44843.2 & 15398.1 & 120.359 & 43.482 & 97.6471 \\
\hline
\end{tabular}

\section{References}

1. C. Bessière and J.-C. Régin. Local consistency on conjunctions of constraints. In Proceedings of the ECAI'98 Workshop on Non-binary constraints, pages 53-59, Brighton, UK, 1998.

2. A. K. Mackworth. On reading sketch maps. In Proceedings of the Fifth International Joint Conference on Artificial Intelligence, pages 598-606, Cambridge, Mass., 1977.

3. J. Gaschnig. Experimental case studies of backtrack vs. Waltz-type vs. new algorithms for satisficing assignment problems. In Proceedings of the Second Canadian Conference on Artificial Intelligence, pages 268-277, Toronto, Ont., 1978.

4. D. Sabin and E. C. Freuder. Contradicting conventional wisdom in constraint satisfaction. In Proceedings of the 11th European Conference on Artificial Intelligence, pages 125-129, Amsterdam, 1994.

5. C. Bessière and J.-C. Régin. Arc consistency for general constraint networks: Preliminary results. In Proceedings of the Sixteenth International Joint Conference on Artificial Intelligence, pages 398-404, Nagoya, Japan, 1997.

6. B. Smith, K. Stergiou, and T. Walsh. Using auxilliary variables and implied constraints to model non-binary problems. In Proceedings of the Seventeenth National Conference on Artificial Intelligence, Austin, Texas, 2000. 